\title{
Visual evoked response in transient monocular visual loss
}

\author{
LANNING B. KLINE' AND JOEL S. GLASER ${ }^{2}$
}

From the 'Eye Foundation Hospital, Department of Ophthalmology, University of Alabama, Birmingham, Alabama, and the ${ }^{2}$ Bascom Palmer Eye Institute, Department of Ophthalmology, University of Miami, Miami, Florida, USA

SUMMARY The pattern-reversal visual evoked response (VER) was recorded in 2 patients during transient monocular visual reduction. In both cases the VER was initially abolished. With recovery of vision there was gradual return of amplitude over a 3- to 8-minute period, while latencies remained unchanged from preattack values. These findings are discussed with regard to current understanding of the origins of the VER and relevant aspects of retinal electrophysiology.

Amaurosis fugax typically is the result of retinal microembolisation, classically from occlusive disease of the extracranial carotid artery, ${ }^{1}$ or from other sources. ${ }^{2-6}$ Rarely, retinal vasospasm has been documented as a cause of transient visual loss. ${ }^{17-9}$ We had the opportunity to record the visual evoked response (VER) during monocular loss and subsequent return of vision in 2 patients. While other aspects of these cases were described elsewhere,$^{89}$ the unique opportunity to record the VER prompted this report. To the best of our knowledge the VER has not been previously recorded during transient monocular visual reduction.

\section{Materials and methods}

During attacks of transient monocular visual loss the VER was obtained in different laboratories. Nevertheless the basic technique was similar, employing pattern-reversal stimulation with large checks $\left(60^{\prime}\right.$ to $80^{\prime}$ of arc) at low frequency $(1-3 \mathrm{~Hz})$, recording transient responses in each case.

Our first patient, wearing appropriate refractive error, was positioned $75 \mathrm{~cm}$ in front of a screen on to which was projected a high-contrast black-and-white checkerboard pattern. The entire pattern subtended $23^{\circ}$ of central visual field with each individual square subtending $60^{\prime}$ of arc. The average luminance at the screen was $270 \mathrm{~cd} / \mathrm{m}^{2}$. Reversal was produced by angular oscillation $(1.2 \mathrm{~Hz})$ of a mirror. Occipital

Correspondence to Dr Lanning B. Kline, Eye Foundation Hospital, 1720 University Boulevard, Birmingham, Alabama 35233, USA. potentials were recorded with bipolar disc electrodes placed in the midline located $1 \mathrm{~cm}$ above the inion and at the vertex; an indifferent electrode was attached to the ear. The signal was passed through a preamplifier (Grass P-15) and a differential amplifier (Tektronix 3A9). Between 64 and 128 responses were averaged (Nicolet 1072 computer-averager) in at least 2 separate runs for each measurement. An observer monitored patient fixation throughout the course of VER testing. This technique produced a VER characterised by a small upward and larger downward deflection at approximately $100 \mathrm{~ms}$. The negative component had the largest amplitude and was the most reliable complex. Latency was measured from the onset of the stimulus to the peak of this major negative component.

VER recordings for the second patient were obtained with a monopolar lead $1 \mathrm{~cm}$ above the inion and with a reference electrode at the mastoid process. The wave forms were recorded with a Nicolet CA1000 system with television pattern generator. Highcontrast black-and-white alternating check stimuli were presented at a reversal rate of $3 \mathrm{~Hz}$, and 128 transient responses were averaged. At a distance of 4 feet $(120 \mathrm{~cm})$ each check subtended $80^{\prime}$ of arc, with the entire screen subtending $10^{\circ} 42^{\prime}$ horizontally and $8^{\circ} 30^{\prime}$ vertically. Patient fixation was monitored continuously by an observer. With this technique the VER was characterised by a small negative deflection and larger positive component at approximately $100 \mathrm{~ms}$. Latency was measured from the onset of the stimulus to the peak of this major upward deflection. 


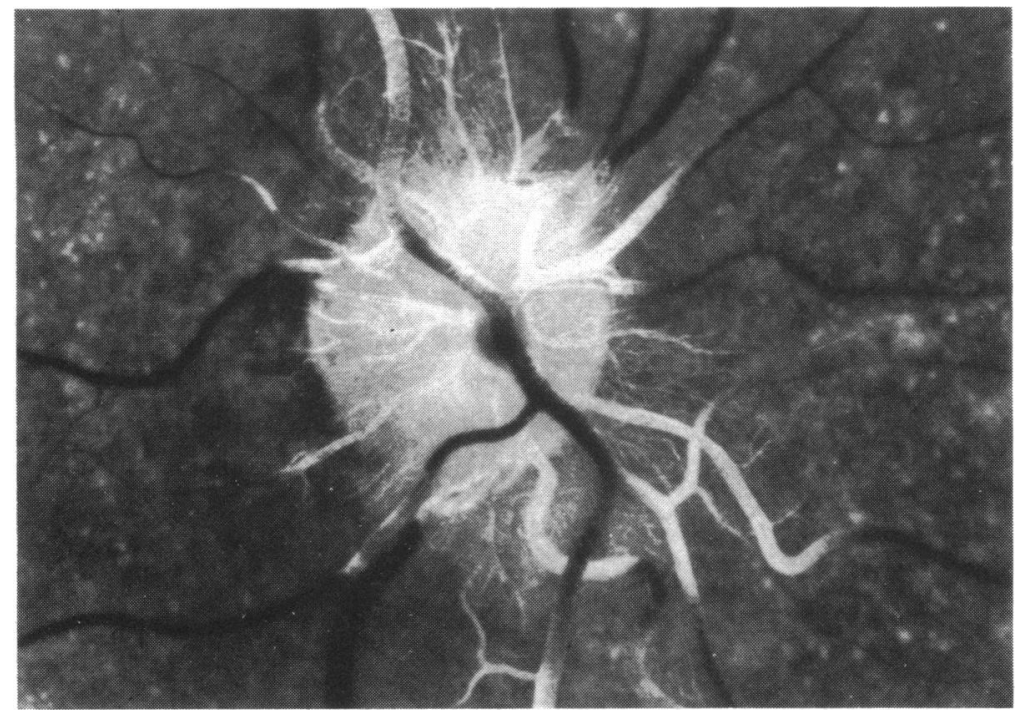

Fig. 1 Case 1. Fluorescein angiography of right fundus, 55 seconds after onset of amaurotic fugax, demonstrates dye within vessels of the optic disc and segmental, incomplete filling of juxtapapillary retinal arterioles and veins. (Reprinted from Shaw et al. $\left.{ }^{8}\right)$.

\section{Case reports and results}

\section{CASE 1}

A 23-year-old black woman had recurrent amaurosis fugax in her right eye for 3 months. When she was initially examined, her visual acuity was 20/20 in each eye. Visual field examination showed a superior arcuate scotoma in the left eye. Ophthalmoscopy revealed dilated arterioles and veins in the right eye. The left fundus showed areas of vascular sheathing suggestive of previous vasculitis. The remainder of the examination was normal.

During examination the patient had repeated amaurotic attacks in the right eye. Right vision was instantaneously reduced to no light perception, with a nonreactive, amaurotic right pupil. Ophthalmoscopy revealed narrowing of retinal arterioles and veins, cessation of retinal blood flow, segmentation of the blood columns, and optic disc pallor. During an episode of transient visual loss fluorescein angiography showed early filling of the right optic disc and peripapillary capillaries, but fluorescein dye was not seen in the retinal arterioles until 30 seconds after injection (Fig. 1). The patient was found to have both SC haemoglobinopathy and systemic lupus erythematosus. After treatment with prednisone the amaurotic attacks abruptly subsided. The patient has remained asymptomatic.

VER was obtained at the onset of amaurosis fugax, and after 2, 5, 8, and 11 minutes (Fig. 2). At the onset of the attack no VER was recordable, and with return of retinal perfusion the VER amplitude gradually increased over a 5-8-minute period. In contrast, as VER amplitudes returned, latencies were unchanged, measuring $94 \mathrm{~ms}$ (normal: $98 \pm 6 \mathrm{~ms}$ ).

CASE 2

Four days prior to examination a 48-year-old man reported the onset of repeated episodes of 'almost

PRE-ATTACK

ONSET OF ATTACK

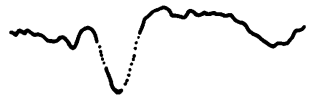

2 MIN. AFTER ONSET

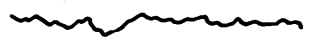

5MIN. AFTER ONSET

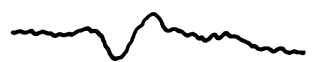

8 MIN. AFTER ONSET

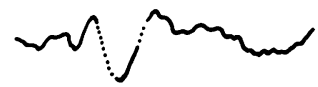

II MIN AFTER ONSET
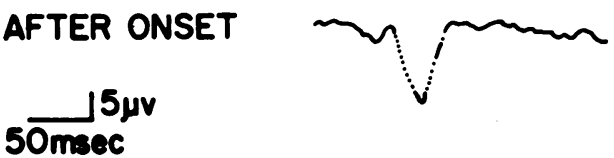

Fig. 2 Case 1. Serial recordings of pattern-reversal VER during an attack of amaurosis fugax on the right due to spasm of the central retinal artery. 
Fig. 3 Case 2. Fluorescein angiography of the left eye 8 seconds after the onset of ocular migraine reveals filling of retinal vessels only inferotemporally. The remainder of the vessels filled approximately 30 seconds later. (Reprinted from Kline and Kelly').

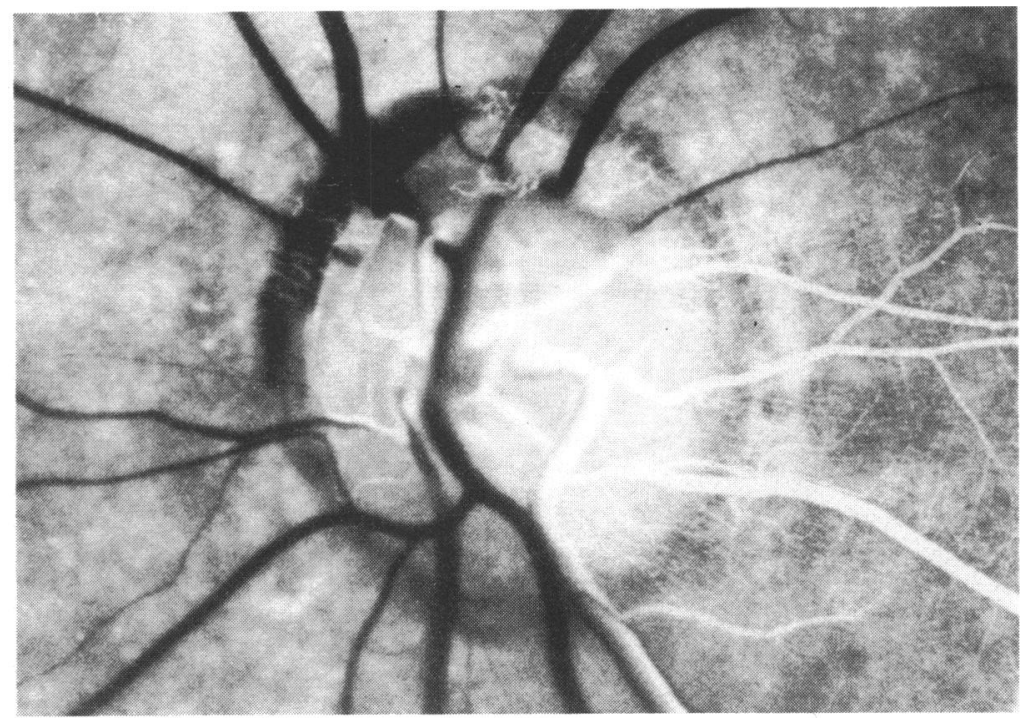

total blindness' of the left eye lasting about 2 minutes. During these episodes he experienced progressive loss of peripheral visual field but maintained a small portion of central visual field, which he described as 'shaped like a leaf.' The patient had experienced classic cluster headaches for the past 25 years but none in the previous 6 months. There was no family history of migraine. While he was asymptomatic,

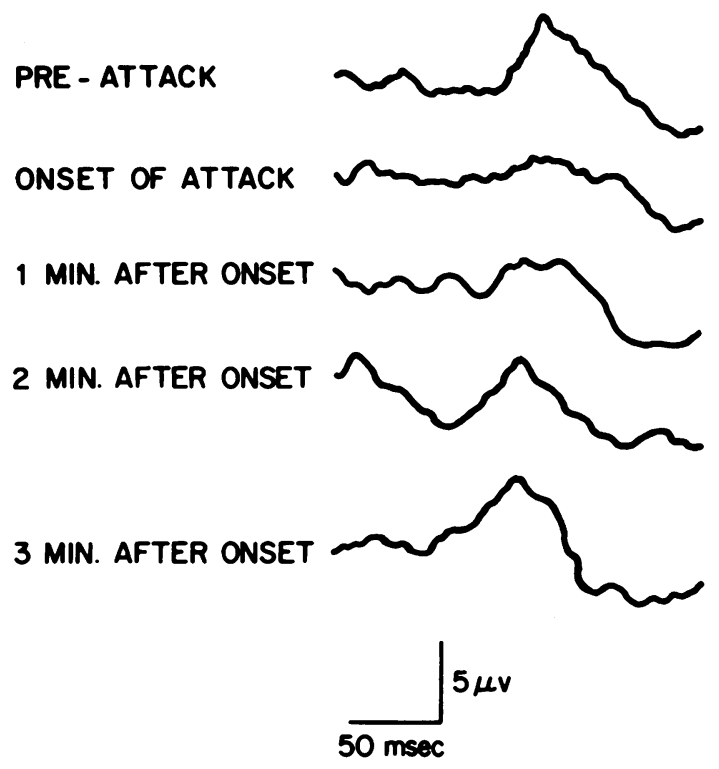

Fig. 4 Case 2. Serial recordings of pattern-reversal VER during an episode of transient visual reduction of the left eye due to ocular migraine. neuro-ophthalmological testing was entirely normal, including funduscopic examination.

While he was in hospital the monocular attacks of visual reduction, each lasting approximately 2 minutes, were studied in detail. The patient maintained visual acuity of $20 / 30$ on the left, and a small central island of visual field. During the attacks the retinal veins narrowed. There was delay in the appearance of fluorescein dye in branches of the central retinal artery yet prompt filling of 2 cilioretinal vessels (Fig. 3). Haematological and cardiac investigations, including echocardiography, gave normal results, and no abnormalities were found on carotid angiography. Diagnosed as having ocular migraine. the patient was placed on propranolol, with prompt cessation of visual symptoms.

As seen in Fig. 4, at the onset of the attack of ocular migraine the VER was abolished. While amplitude returned over a 2-3-minute period, the latencies (102 ms) were essentially unchanged from preattack values (normal: $99 \mathrm{~ms} \pm 7$ ).

\section{Discussion}

The series of electrical and electrochemical events that leads to the elaboration of the VER begins in the photoreceptors of the retina and culiminates in the occipital cortex. Pattern-reversal VER has been found to show a more consistent waveform and to be more sensitive in detecting lesions of the visual pathways than has flash response. ${ }^{10}$ In the assessment of afferent input to the visual cortex pattern-reversal VER has been studied in a variety of diseases of the optic nerve, including optic neuritis, ${ }^{11}$ ischaemic optic 
neuropathy,,$^{12}{ }^{13}$ toxic amblyopia, ${ }^{14}$ glaucoma,,${ }^{15}$ and compressive optic neuropathy. ${ }^{16}$ Comparison of amplitude and latency abnormalities has at times distinguished among such optic nerve diseases. For example, in the acute phase of demyelinative optic neuritis the amplitude of the pattern-reversal VER is reduced, and it returns to normal with clinical recovery, while characteristically prolonged latency remains. ${ }^{11}$ However, in many instances it is difficult to separate the relative contribution of axonal degeneration and demyelination when VER abnormalities occur.

The 2 cases presented here provide an opportunity to examine selective impairment of axonal transmission within the optic nerve without disturbance of myelin sheaths. The central retinal artery is the sole vascular supply of the inner retinal layers (ganglion cells, inner plexiform layer, inner nuclear layer) ${ }^{17}$ With temporary interruption of blood flow, inner retinal activity ceases and no VER is generated. With resumption of blood flow there is progressive increases in retinal neuronal function, and VER amplitude gradually returns (Figs. 2 and 4). But with gradual return of inner retinal function there is nevertheless a constant value for VER latency.

Investigations into the genesis of the VER have dealt with contributions from striate (area 17) and extrastriate (areas 18 and 19) cortex. ${ }^{18-20}$ An understanding of the relationship between the VER and underlying neural events is far from complete. The only study dealing with the origin of the transient pattern reversal VER was done by Halliday and Michael ${ }^{18}$ who found that the largest amplitude responses were recorded at electrodes located 5 to $7.5 \mathrm{~cm}$ in front of the inion, a site anterior to the striate cortex. They concluded that the major deflection occurring at approximately $100 \mathrm{~ms}$ is generated in extrastriate cortex.

Our studies demonstrate that return of inner retinal function, with no damage to optic nerve myelin, causes no alteration in VER latency. This phenomenon suggests that the inner retina responds in an all-or-none fashion. Current understanding of retinal electrophysiology supports this interpretation. With visual excitation the majority of retinal neural cells respond in a slowly graded manner, with the exception of retinal ganglion cells and some amacrine cells, where depolarisation leads to an all-or-none action potential. ${ }^{2122}$ With interruption and subsequent return of inner retinal activity there is a gradual and progressive return of VER amplitude, presumably reflecting the number of functioning ganglion cells. yet an immediate restitution of latency occurs, possibly due to the all-or-none action potentials of the retinal ganglion cells.

\section{References}

1 Walsh FB. Hoyt WF. Clinical Neuro-ophthalmology. 3rd ed. Baltimore: Williams and Wilkins, 1969: 1671-2, 1806.

2 Penner R, Font RL. Retinal embolism from calcified vegetations of aortic valve. Arch Ophthalmol 1969; 81: 565-8.

3 Zimmerman LE. Embolism of the central retinal artery. Arch Ophthalmol 1965; 73: 822-6.

4 Kearns TP. Fat ambolism of the retina. Am J Ophthalmol 1956; 41: $1-2$.

5 Manschot WA. Embolism of the central retinal artery. Am J Ophthalmol 1959; 48: 381-5.

6 Fischbein FI. Ischemic retinopathy following amniotic fluid embolization. Am J Ophthalmol 1969; 67: 351-7.

7 Carpenter WM, Carpenter EW. Raynaud's disease with intermittent spasm of the retinal artery and veins. Arch Ophthalmol 1938; 19: 111-3.

8 Shaw HE, Osher RH, Smith JL. Amaurosis fugax associated with SC hemoglobinopathy and lupus erythematosus. Am J Ophthalmol 1979; 87: 281-5.

9 Kline LB, Kelly CL. Ocular migraine in a patient with cluster headaches. Headache 1980; 20 : 253-7.

10 Halliday AM. Clinical application of evoked potentials. In: Mathews WB, Glaser GH. Recent Advances in Clinical Neurology. New York: Churchill, 1978: 47-73.

11 Halliday AM, McDonald WI, Mushin J. Delayed visual evoked potential in optic neuritis. Lancet 1972; i: 982-5.

12 Wilson WB. Visual evoked response: differentiation of ischemic optic neuritis from the optic neuritis of multiple sclerosis. Am J Ophthalmol 1978; 86: 530-5.

13 Glaser JS, Laflamme P. The visual evoked response: methodology and application in optic nerve disease. In: Thompson HS, ed. Five Topics in Neuro-ophthalmology. Baltimore: Williams and Wilkins, 1979: 199-218.

14 Halliday AM. Visually evoked responses in optic nerve disease. Trans Ophthalmol Soc UK 1976; 96: 372-6.

15 Cappin JM, Nissum S. Visual evoked responses in the measurement of field defects in glaucoma. Arch Ophthalmol 1975; 93: 9-18.

16 Halliday AM, HAlliday E, Kriss A, McDonald WI, Mushlin J. The pattern-evoked potential in compression of the anterior visual pathways. Brain 1976; 99: 357-4.

17 Duke-Elder S. System of Ophthalmology. St Louis: Mosby, 1961: 2: 230-7.

18 Halliday AM, Michael WE. Changes in pattern-evoked responses in man associated with the vertical and horizontal meridians of the visual field. J Physiol 1970; 208: 499-513.

19 Jeffreys DA, Axford JG. Source location of pattern-specific components of human visual evoked potentials. I. Components of striate cortical origin. Exp Brain Res 1972; 16: 1-21.

20 Jeffreys DA, Axford JG. Source location of pattern-specific components of human visual evoked potentials. II. Components of extrastriate cortical origin. Exp Brain Res 1972; 16: 22-40. .

21 Witkovsky P. Peripheral mechanisms of vision. Annu Rev Physiol 1971; 33: 257-80.

22 Davson $\mathrm{H}$. The Eye. Visual Function in Man. New York: Academic Press, 1976: 2A: 291. 